\title{
Natural cytotoxicity receptor 1 in mouse uNK cell maturation and function
}

\author{
AM Felker ${ }^{1}$ and BA Croy ${ }^{1}$
}

Early and midgestational decidua of mice genetically ablated for expression of the natural killer (NK) cell natural cytotoxicity receptor (NCR; $N$ cr1 $1^{G f p / G f p}$ mice) shows restricted angiogenesis and atypically small uterine (u)NK cells. We hypothesized that NCR1 inactivation disturbs maturation and angiokine production by uterine natural killer (UNK) cells. Using histological and morphometric approaches, we observed that Ncr1 ${ }^{\text {Gfp/Gfp }}$ but not control C57BL/6 (B6) implantation sites sustain immature, non-granulated UNK cells into midpregnancy. Mouse uNK cells can be subclassified by their reactivity with Dolichos biflorus agglutinin (DBA) lectin; DBA + uNK cells with greater Ncr1 expression were investigated. DBA + uNK cells from $N c r 1^{G f p / G f p}$ mice show delayed maturation as indicated by shorter diameters and fewer cytoplasmic granules. Granules in mature $N c r 1^{\text {Gfp/Gfp }}$ uNK cells are ultrastructurally abnormal and abundance of granule-associated proteins (perforin, granzyme) and of cytoplasmic proteins (vascular endothelial growth factor; placental growth factor) differs from controls. Leukocyte-leukocyte conjugate formation in gestation day 6.5 and 8.5 intact $N c r 1^{\text {Gfp/Gfp }}$ decidua was less frequent than in B6; however, this difference involved leukocytes other than DBA + uNK cells. These studies strongly support roles for NCR1 and its ligands in normal pregnancy promotion.

\section{INTRODUCTION}

Successful human and mouse pregnancy involves contributions from large, multilineage decidual leukocyte populations. Dominant in early decidua basalis (DB) are proliferative uterine natural killer (uNK) cells. ${ }^{1,2}$ Human uNK cells are globally identified by their $\mathrm{CD} 56^{\text {bright }} \mathrm{CD} 16^{-}$surface phenotype. ${ }^{3}$ In mice, two major uNK cell subsets are histologically distinguished by reactivity with Dolichos biflorus agglutinin (DBA) lectin. ${ }^{4}$ Most DBA- uNK cells (72\%) co-express Ly49C/I and NK1.1. ${ }^{1}$ Neither DBA + nor DBAuNK cells use the transcription factor NFIL3 (E4BP4) for differentiation, a requirement for peripheral NK (pNK) cells. ${ }^{5,6}$ Mature uNK cells (DBA + and DBA-) contain large numbers of cytoplasmic granules with contents that include perforin (PRF) and granzyme A (GZMA), ${ }^{7-10}$ but uNK cells have limited cytotoxicity in vitro. ${ }^{10}$ In human uNK cells, failed microtubular assembly has been documented to prevent polarization of PRF-containing granules during immune synapse formation. ${ }^{11}$ Mouse and human uNK cells are recognized as important producers of cytokines and angiokines within $\mathrm{DB}^{3,8,12-14}$ with functional products differing between DBA + and DBA- uNK cell subsets. ${ }^{12,13}$

As in pNK cells, uNK cells express numerous activating and inhibitory receptors that drive their functions. Human uNK cells constitutively express killer immunoglobulin-like receptors that interact with major histocompatibility complex (MHC) molecules expressed by invading trophoblast cells. ${ }^{15} \mathrm{In}$ mice, uNK cells utilize LY49 lectin receptors to recognize MHC. LY49 receptor family usage contributes to uNK cell granule biogenesis, synthesis of vascular endothelial growth factor (VEGF), early decidual angiogenesis, and midgestational, uNK cell-initiated, spiral arterial remodeling. ${ }^{16}$

Other NK cell receptors bind non-MHC ligands. Natural cytotoxicity receptors (NCRs) are expressed by human and mouse pNK and uNK cells. In humans, pNK and uNK cells express different splice variants of NKp46/NCR1, NKp44/NCR2, and NKp30/NCR3 and the altered NKp30 and NKp44 receptor isoform profile of uNK cells is associated with decreased cytotoxicity, secretome modifications, and impaired immune synapse formation. ${ }^{17} \mathrm{NCR} 1$, the murine NKp46 homolog, ${ }^{18}$ is expressed on $\mathrm{pNK}$ and uNK cells, ${ }^{19}$ with dramatically higher expression by DBA + than by DBA- uNK cells. ${ }^{20}$

NCRs have multiple roles in non-pregnant humans and mice. In humans, NCRs are essential for in vitro lysis of cancer cell lines ${ }^{21}$ and recognition of herpes simplex virus-infected cells. $^{22}$ In mice, NCR1 is implicated in influenza virus

${ }^{1}$ Department of Biomedical and Molecular Sciences, Queen's University, Kingston, Ontario, Canada. Correspondence: AM Felker, a.felker@queensu.ca Received 10 May 2016; accepted 29 November 2016; published online 18 January 2017. doi:10.1038/mi.2016.126 
Gd6.5

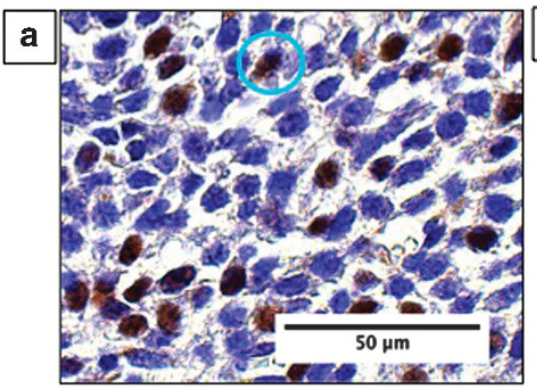

Gd10.5
Gd8.5

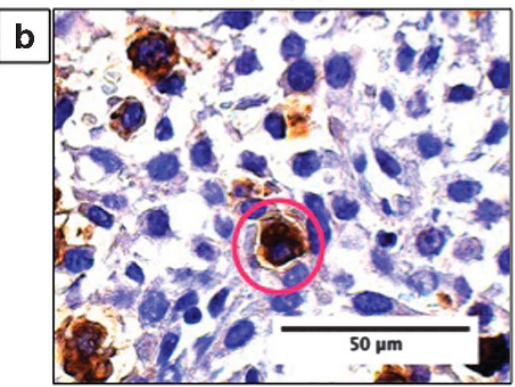

Gd12.5
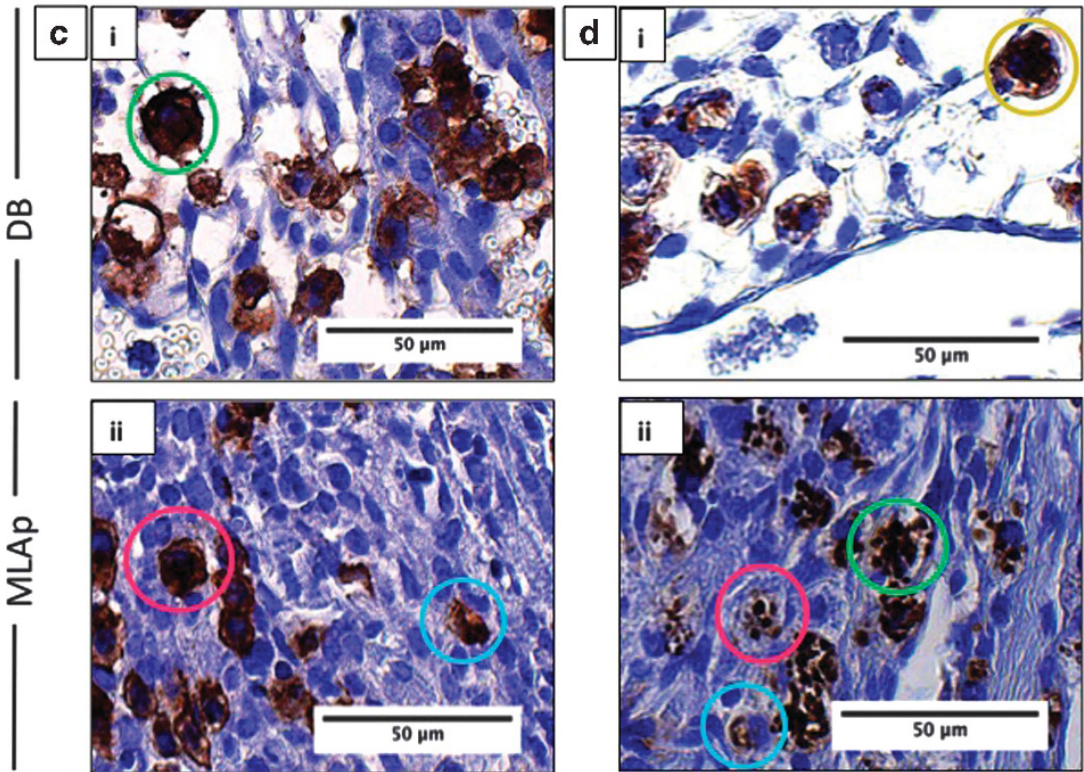

Figure 1 Maturation subtypes of DBA $+N c r 1^{G f p / G f}$ uNK cells. Four distinct stages of uNK cell maturation are shown at gd6.5 (a), 8.5 (b), 10.5 (c), and

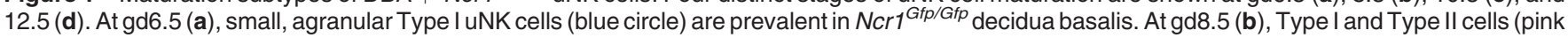
circle) are present in high proportions. At gd10.5 (c), the DB (i) and MLAp (ii) are distinct regions with uNK cells in the MLAp typically less mature than in DB. Type III uNK cells (green circle) are observed in DB (i), but are absent from the MLAp (ii) where Type I (blue circle) and Type II (pink circle) are present. At gd12.5 (d), Type I cells persist in Ncr ${ }^{\text {Gfp/Gfp }}$ decidua basalis (i) and Type IV uNK cells (yellow circle) are observed for the first time. In gd12.5 MLAp (ii), only Type I-III uNK cells occur (circled). Images are representative of three separate staining series using two implantation sites from three different pregnancies. DBA, dolichos biflorus agglutinin; DB, decidua basalis; gd, gestation day; GFP, green fluorescent protein; MLAp, mesometrial lymphoid aggregate of pregnancy; NCR1, natural cytotoxicity receptor; uNK, uterine natural killer.

clearance ${ }^{23}$ and in intestinal mucosal defense. ${ }^{24}$ In both species, NKp46/NCR1 is implicated in development of type 1 diabetes through recognition of ligands on and subsequent killing of pancreatic $\beta$ cells. ${ }^{25}$

Human clinical investigations identified NKp46 + uNK cell in decidual cell suspensions from mid-secretory phase endometrial biopsies of women experiencing recurrent pregnancy loss. ${ }^{26}$ Others report NKp46+ pNK cells are less frequent in human pregnancies complicated by gestational diabetes. ${ }^{27}$ In vitro studies of first trimester human uNK cells also found that antibody-dependent engagement of NKp46 induced PRF polarization and degranulation; ${ }^{17,28}$ cytotoxicity, however, was blocked by co-engagement of the uNK cell inhibitory receptor NKG2A. ${ }^{28}$ NKp46 cross-linking of first trimester human uNK cells also induces dose-dependent production of cytokines that include VEGF and placental growth factor (PGF). ${ }^{8,17}$
Only one report has addressed NCR1 contributions during mouse pregnancy using an Ncrl-deficient mouse with a green fluorescent protein (GFP) reporter cassette replacing $\mathrm{Ncr} 1$ (MAR-1) ${ }^{18,23}$ Ncr1 loss delayed conceptus growth and elevated fetal resorption frequency. ${ }^{20}$ Histological and whole-mount in situ immunohistochemical (WM-IHC) studies of these Ncr1deficient implantation sites also identified smaller uNK cells, impaired decidual angiogenesis, and delayed spiral arterial remodeling. ${ }^{20}$ Here we address the roles of NCR1 in uNK subtype differentiation, cytoplasmic granule and angiokine synthesis, and $\mathrm{DBA}+\mathrm{uNK}$ cell conjugate formation in early decidua.

\section{RESULTS}

Fecundity and fertility in $\mathrm{Ncr} 1^{\text {Gfp/Gfp }}$ mice

$N c r 1^{G f p / G f p} \times N c r 1^{G f p / G f p}$ breeding pairs housed at Queen's University, produced an average of 5.1 pups per litter, with a 
Table 1. DBA + uNK cell maturation subtypes and lytic protein expression

\begin{tabular}{|c|c|c|c|c|c|c|c|c|c|c|c|c|}
\hline & \multicolumn{6}{|c|}{ C57BL/6 } & \multicolumn{6}{|c|}{$\mathrm{Ncr} 1^{\text {Gfp/Gfp }}$} \\
\hline & Gd6.5 & Gd8.5 & DB & MLAp & DB & MLAp & Gd6.5 & Gd8.5 & DB & MLAp & DB & MLAp \\
\hline \multicolumn{13}{|c|}{ Percent DBA + UNK cells at different maturation stages } \\
\hline Type I & 72.9 & 66.9 & 18.4 & $50.6^{\mathrm{a}}$ & 0 & 0 & $83.2^{\mathrm{b}}$ & 71.8 & 20.9 & $62.3^{\mathrm{a}}$ & $7.2^{\mathrm{b}}$ & $16.2^{\mathrm{b}}$ \\
\hline Type ॥ & 27.1 & 30.9 & 46.0 & 47.3 & 29.1 & $46.7^{\mathrm{a}}$ & $16.8^{\mathrm{b}}$ & 26.3 & $54.4^{b}$ & $37.3^{a}$ & $36.8^{\mathrm{b}}$ & 50.4 \\
\hline Type IV & 0 & 0 & 0 & 0 & 17.0 & $0^{\mathrm{a}}$ & 0 & 0 & 0 & 0 & 7.0 & $0^{\mathrm{a}}$ \\
\hline \multicolumn{13}{|c|}{ Perforin and granzyme A expression } \\
\hline Perforin and granzyme A & 63.4 & 62.6 & 76.7 & $18.8^{\mathrm{a}}$ & 69.0 & $48.7^{\mathrm{a}}$ & $37.6^{\mathrm{b}}$ & $45.8^{\mathrm{b}}$ & $58.5^{\mathrm{b}}$ & $24.8^{\mathrm{a}}$ & 58.0 & 44.7 \\
\hline Perforin alone & 13.2 & 8.3 & 14.4 & $39.8^{\mathrm{a}}$ & 19.7 & 25.3 & 13.9 & $27.1^{\mathrm{b}}$ & $27.8^{\mathrm{b}}$ & 40.3 & 17.0 & 27.3 \\
\hline
\end{tabular}

male:female pup ratio of 1.04:1 ( $n=41$ live-born litters). In $14.6 \%$ of litters, pups were found dead shortly after parturition. For $N c r 1^{G f p / G f p}$ females killed during pregnancy $(n=51)$, the average pups per pregnancy was 8.1 equally distributed between right and left uterine horns. The rate of sterile mating (i.e., copulation plug but no pregnancy) in $N c r 1^{G f p / G f p}$ females was $25.5 \%$. These are in line with values previously reported ${ }^{16}$ and observed in our facilities for B6 mice. Fetal resorptions occurred in $26.3 \%$ of $N c r 1^{G f p / G f p}$ pregnancies $(n=38)$ and represented $10.6 \%$ of all implant sites $(n=333)$, rates higher than in our B6 pregnancies (resorptions in $15.0 \%$ of pregnancies $(n=20)$; $2.4 \%$ of all implant sites $(n=165))$.

\section{Delayed uNK cell maturation in Ncr1 ${ }^{\text {Gfp/Gfp }}$ mice}

Previous studies found that Ncrl loss did not alter uNK cell numbers or DBA + /DBA - uNK cell proportions between gestation day (gd)6.5 and 12.5, but identified smaller uNK cells at these gd. ${ }^{20}$ To determine if smaller $N c r 1^{\text {Gfp/Gfp }}$ uNK cells reflected delayed maturation, maturation subtypes I-IV of DBA + uNK cells residing in the decidua (Supplementary Figure S1 online) were scored using morphologically defined criteria (diameter, number of cytoplasmic granules, and nuclear morphology (Figure 1)). ${ }^{4,6}$ At gd6.5, most DBA + uNK cells in B6 and Ncr1 $1^{\text {Gfp/Gfp }}$ mice were small, agranular Type I uNK cells (Figure 1a). A significantly smaller proportion of cells in both B6 and Ncr1 ${ }^{\text {Gfp/Gfp }}$ mice were Type II uNK cells with slightly larger diameters and few DBA + cytoplasmic granules. No Type III or IV cells were present in gd6.5 decidua in either strain. The proportion of Type I uNK cells was significantly higher in $N c r 1^{G f p / G f p}$ compared with B6 mice $(P=0.047)$ and there was a relative deficit in the proportion of Type II uNK cells in $N c r 1^{\text {Gfp/Gfp }}$ compared with B6 (Table $1 ; P=0.047$ ). At gd8.5, Type I and Type II uNK cells were present in high numbers (Figure 1b), heavily granulated Type III uNK cells were observed but senescent Type IV cells were absent in both genotypes. There were no significant differences between B6 and $N c r 1^{G f p / G f p}$ mice in the proportions of Type I $(P=0.379)$, Type II $(P=0.412)$, or Type III $(P=0.553)$ uNK cells at gd 8.5 (Table 1).

By gd10.5, the MLAp is a fully differentiated region distinct from DB (Supplementary Figure S1). The MLAp contains different proportions of uNK cell subtypes than DB (Figure 1c). At gd10.5, gd $N c r 1^{G f / p / G f p}$ and B6 DB mice had similar proportions of Type I uNK cells $(P=0.505$ but a higher frequency of Type II $(P=0.033)$ and slightly diminished frequency of Type III uNK cells $(P=0.054)$ persisted in Ncr1 ${ }^{G f p / G f p}$ mice (Table 1). MLAp typically has a higher proportion of immature uNK cells than DB (Figure 1c). This was observed at gd10.5 with higher proportions of Type I uNK cells in B6 and Ncr1 ${ }^{\text {Gfp/Gfp }}$ MLAp (Table 1). In B6, similar proportions of Type II uNK cells were present in the MLAp and DB $(P=0.849)$, but Type III cell proportions were lower in MLAp vs. DB $(P<0.001)$. Type IV cells were absent from both regions. In gd10.5 Ncr1 $1^{G f p / G f p}$, Type II $(P=0.015)$ and Type III $(P=0.003)$ uNK cell subtypes were less frequent in the MLAp (Table 1; Figure 1c). There were no significant gd10.5 differences in uNK cell subtype proportions in the MLAp (Table 1).

In gd12.5 DB Type I uNK cells were absent in B6 but persisted in Ncrl Gfp/Gfp $(P<0.001)$ (Table 1). The Type II uNK cell proportion was also greater in DB of $N c r 1^{\text {Gfp/Gfp }}$ than B6 $(P=0.009)$ but Type III uNK cell frequencies were similar $(P=0.285)$. Fully mature Type IV uNK cells, which are normally first observed at gd12.5 (Figure 1d), tended to be less frequent in $N c r 1^{G f p / G f P} \mathrm{DB}(P=0.063)$ (Table 1). Gd12.5 uNK cells remained more immature in the MLAp than DB for both genotypes (Figure 1d). Type I uNK cells were absent from B6 MLAp but present in Ncr $1^{\text {Gfp/Gfp }}$ MLAp. Type II uNK cells were more frequent in MLAp than DB in B6 $(P=0.014)$ and Ncr1 ${ }^{G f p / G f p}$ mice $(P=0.053)$, whereas Type III uNK cell 
frequencies did not differ significantly between the MLAp and DB in either B6 $(P=0.927)$ or $N c r 1^{G f p / G f p}(P=0.085)$ (Table 1). Type IV cells were not detected in the MLAp of either B6 or $N c r 1^{G f p / G f p}$ (Figure 1d). Compared with B6, Ncr1 ${ }^{G f p / G f p}$ had significantly more Type I $(P=0.004)$ and fewer Type III $(P=0.052)$ uNK cells in the MLAp, whereas Type II cells were not significantly different $(P=0.653)$ (Table 1).

As higher proportions of immature uNK cell subtypes were observed in $N c r 1^{G f p / G f p}$ mice, we asked if programmed cell death that typically begins $\sim$ gd12.5 (ref. 29 ) is delayed in $N c r 1^{G f p / G f p}$. TUNEL staining of B6 and Ncr1 ${ }^{G f p / G f p}$ gd10.5 and 12.5 implantation sites revealed very little evidence of apoptosis in nucleated decidual cells, including uNK cells, of either strain (data not shown).

\section{uNK cell granulogenesis and lytic protein expression are impaired in $\mathrm{Ncr} 1^{\text {Gfp/Gfp }}$ mice}

Acquisition of cytoplasmic granules that contain lytic proteins characterizes uNK cell maturation. ${ }^{4,9}$ Ultrastructural analyses of B6 and $\mathrm{Ncr} 1^{\mathrm{Gfp} / \mathrm{Gfp}}$ decidua at gd6.5 revealed low numbers of uNK cells containing round, immature cytoplasmic granules. The granules were markedly smaller in $\mathrm{N}_{\mathrm{A}} 1^{\mathrm{Gfp} / \mathrm{G} f \mathrm{p}}$ uNK cells (Figure 2b). By gd8.5, granules were more abundant but remained smaller and more immature in $N c r 1^{G f p / G f p}$ than in B6 (Figure 2d). By gd10.5, B6 uNK cell granules had acquired their classical mature phenotype; i.e., round, relatively large, and showing a secretory-lysosome double-compartment morphology with a defined cortical cap and solid, uniformly electron dense core $^{30}$ (Figure 2e). In contrast, $N c r 1^{G f p / G f p}$ uNK cell granules displayed abnormal shapes and many lacked the double compartment phenotype or had anomalous, variable core electron density (Figure 2f).

To determine if impaired cytoplasmic granule development in $N c r 1^{G f p / G f p}$ uNK cells altered PRF or GZMA expression, immunohistochemistry was undertaken to assess lytic protein expression in DBA + uNK cells between gd6.5 and 12.5. Importantly, numbers of $\mathrm{DBA}+$ granules expressed by matched uNK cell subtypes were similar between the strains at each gd (data not shown). At gd6.5 when most DBA + uNK cells are Type I agranular cells (see above), $63.4 \pm 9.9 \%$ of B6 and $37.6 \pm 6.1 \%$ of $N_{c r 1} 1^{G f p / G f p} \mathrm{DBA}+$ uNK cells diffusely expressed both PRF and GZMA $(P=0.018)$. An additional $13.2 \pm 7.9 \%$ of $\mathrm{B} 6$ and $13.9 \pm 4.3 \%$ of $\mathrm{Ncrl}^{\mathrm{Gfp} / G f p}$ of $\mathrm{DBA}+$ uNK cells expressed PRF without GZMA reactivity $(P=0.893)$. When both dual and single expression was considered, total $N c r 1^{G f p / G f p}$ uNK cells expressing lytic protein were diminished vs. B6 $(P=0.026)$ (Table 1). Neither genotype at gd6.5 nor later studied gd had DBA + uNK cells solely reactive for GZMA, whereas some DBA + uNK cells were nonreactive for both PRF and GZMA at all gd (Figure 3).

By gd8.5, Type II and III uNK cells (evident granules) had become more abundant and cytolytic protein expression was less diffuse and more localized to DBA + granules (Figure $3 \mathbf{b}$ ). Gd8.5 Ncr ${ }^{G f p / G f p}$ continued to have fewer dually expressing $\mathrm{DBA}+\mathrm{uNK}$ cells than $\mathrm{B} 6(P=0.003)$ but their proportion of PRF only reactivity was significantly higher $(P<0.001)$. The total proportion of gd8.5 DBA + uNK cells expressing lytic proteins did not differ between genotypes $(P=0.551)$ (Table 1).

At gd10.5 when DB and MLAp are distinct regions (Figure 3c; Supplementary Figure S1), the proportion of $\mathrm{DBA}+\mathrm{uNK}$ cells in DB co-expressing PRF and GZMA remained lower in $N c r 1^{G f p / G f p}$ than B6 $(P=0.038)$ and the statistical difference in sole PRF expression was sustained $(P=0.042)$. DBA + uNK cells expressing both PRF and GZMA were less frequent in the MLAp than in DB of both B6 $(P=0.001)$ and $N c r 1^{G f p / G f p-}(P<0.001)$, whereas the proportion of DBA + uNK cells expressing only PRF was greater in the MLAp than DB in both strains (Table 1). Between strains in MLAp, no significant differences were observed in proportions of dually $(P=0.296)$ or singly $(P=0.964)$ expressing DBA + uNK cells (Table $\mathbf{1}$ ).

In gd12.5 DB, DBA + uNK cell expression of both lytic proteins $(P=0.076)$ or sole PRF expression $(P=0.341)$ did not differ between $N_{c r 1} 1^{G f p / G f p}$ and B6 uNK cells (Table 1; Figure 1d). The proportion of dually positive uNK cells continued to be lower in the MLAp than DB in both strains, whereas the proportion of singly positive uNK cells did not differ significantly (Table 1). No differences were observed in gd12.5 B6 and Ncr1 Gfp/Gfp MLAp regarding either dually $(P=0.649)$ or singly $(P=0.693)$ positive uNK cells. Thus, the morphological alterations in $N c r 1^{G f p / G f p}$ granule biogenesis are accompanied by a lower frequency of granule-contained cytolytic proteins by DBA + uNK cells.

\section{Angiokine expression is altered in Ncr1 $1^{\text {Gfp/Gfp }}$ decidua and DBA + UNK cells}

In normal mice, VEGF is secreted from cytoplasmic vesicles of agranular (Type I) and granular (Types II-IV) DBA + uNK cells. ${ }^{9}$ PGF is also a major angiokine produced by uNK cells. To determine if the deficit in NCR1 signaling impacted production of molecules not stored in secretory-lysosomal granules, time course, quantitative immunohistochemical studies of VEGF and PGF reactivity in DBA + uNK cells and total DB were undertaken. At gd6.5 when DBA + uNK cells are rare, few VEGF-reactive cells were present in $N_{c r} 1^{G f p / G f p}$ and B6 implant sites. Analysis of VEGF fluorescence intensity within the whole decidua revealed elevated VEGF expression in $N c r 1^{G f p / G f p}$ compared with B6 implant sites $(P<0.0001) \quad$ (Supplementary Figure S2). Greater VEGF intensity was also observed in whole decidua of $N c r 1^{\text {Gfp/GfP }}$ mice compared with B6 at gd8.5 $(P<0.001), 10.5(P<0.001)$, and $12.5(P=0.016)$ (data not shown). At gd8.5, when DBA + UNK cell numbers have increased, VEGF expression within $\mathrm{DBA}+$ uNK cells could be measured in addition to whole decidua. Higher VEGF levels were present in gd8.5 $N c r 1^{\text {Gfp/Gfp }}$ DBA + uNK cells than B6 $(P=0.021)$. The difference in VEGF immunoreactivity of uNK cells was more pronounced at gd10.5 $(P=0.011)$ but had equalized by gd12.5 $(P=0.175)$ (Figure $4 a-c)$.

PGF expression within gd6.5 decidua did not differ between B6 and $N c r 1^{\mathrm{G} f p / G f p}$ implant sites $(P=0.331)$ (Supplementary Figure S2), however less total PGF expression was observed 

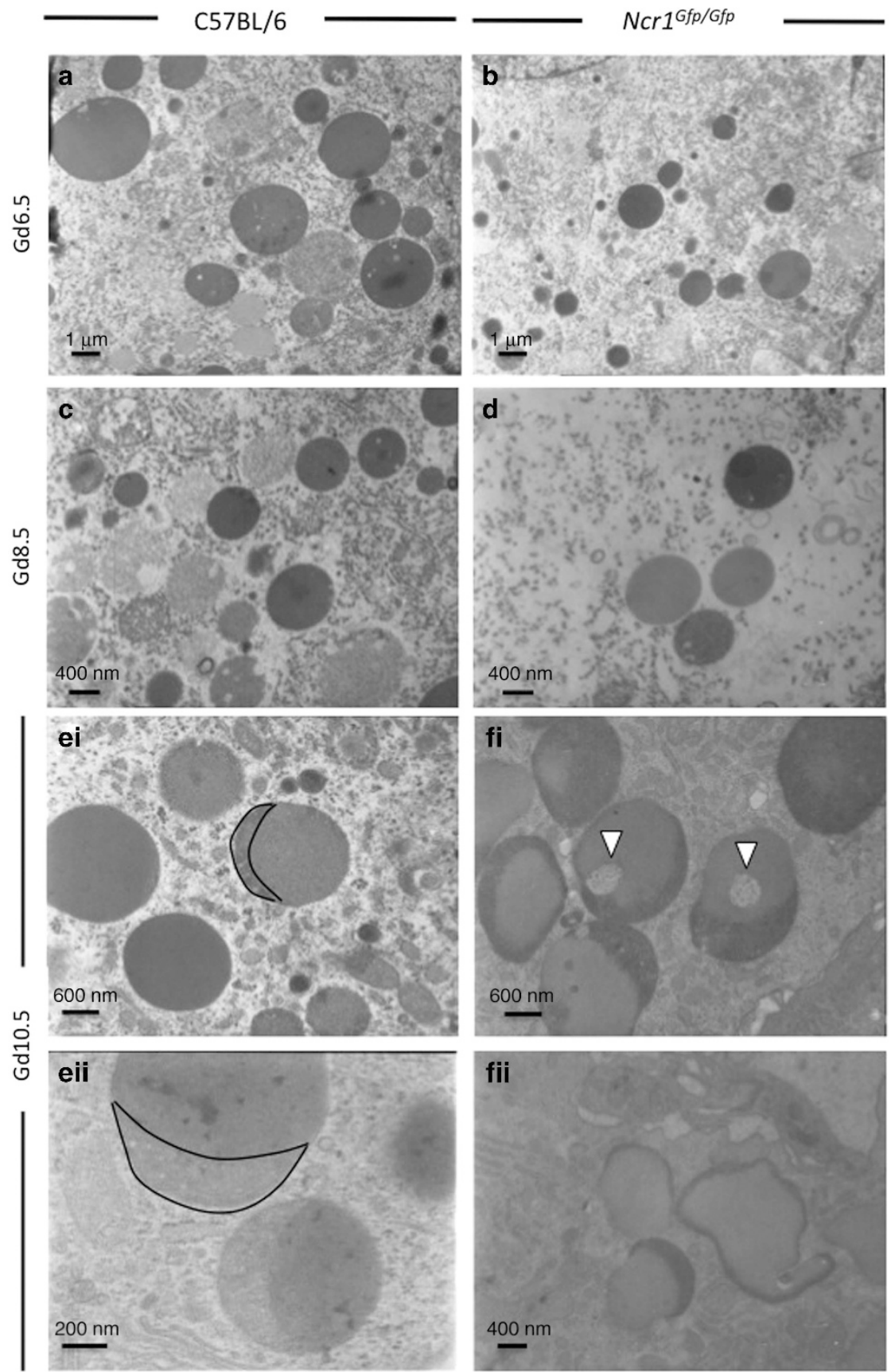

Figure 2 Gd6.5-10.5 C57BL/6 (B6) and Ncr1 ${ }^{\text {Gfp/Gfp }}$ uNK cell granule ultrastructure. Granules in gd6.5 B6 (a) and Ncr1 Gfp/Gfp (b) uNK cells are infrequent, round, and immature. At gd8.5 (c, d) granules increase in frequency but remain immature in B6 (c) and Ncr1 $1^{\text {Gfp/Gfp }}$ (d) uNK cell cytoplasm. At gd10.5 (e,f), B6 uNK cell granules (e) are mature. They remain round but are larger with a well-defined cortical membrane cap and a solid, electron dense core (outlined in ei and at higher power in eii). In gd10.5 Ncr1 ${ }^{\text {Gfp/Gfp }}$ uNK cells (f), granules are structurally variable with many having anomalous electron light core regions (fi; arrowheads), and irregular shapes with atypical cortical caps (fii). Images are representative of ultrastructure studies conducted on two implantation sites from three different pregnancies per gd per genotype. gd, gestation day; GFP, green fluorescent protein; NCR1, natural cytotoxicity receptor; uNK, uterine natural killer.

within $N_{c r 1} 1^{G f p / G f p}$ decidua than B6 at gd8.5 $(P=0.01), 10.5$ $(P=0.029)$, and $12.5(P<0.001)$ (data not shown). When gd8.5 $\mathrm{DBA}+\mathrm{uNK}$ cells were assessed for PGF expression, significantly less PGF reactivity was detected in $N c r 1^{G / p / G f p}$ than $B 6(P=0.002)$ This trend continued at gd10.5 $(P=0.034)$. At gd12.5, in contrast to total decidua, PGF levels in DBA + uNK cells normalized between genotypes $(P=0.187)$ (Figure $4 \mathbf{b})$. Thus, Ncrl gene inactivation inversely dysregulates VEGF and PGF expression in decidua and within uNK cells.

\section{NCR1 deficiency alters conjugation of leukocytes in early (gd6.5 and 8.5) DB}

Overall leukocyte frequencies in $\mathrm{Ncr} 1^{\text {Gtp/Gtp }}$ mice. $\mathrm{DBA}+$ and DBA- (nearly fully defined $(72 \%)$ as Ly49C/I + or NK1.1+) 
Gd6.5

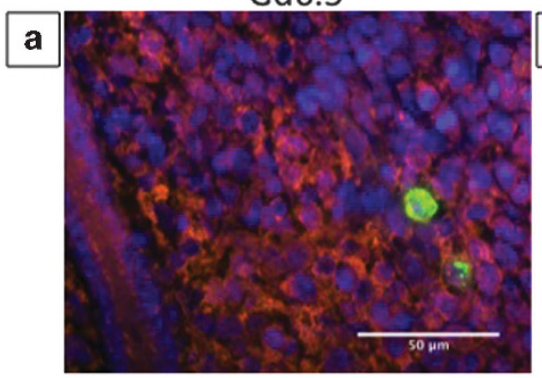

Gd10.5
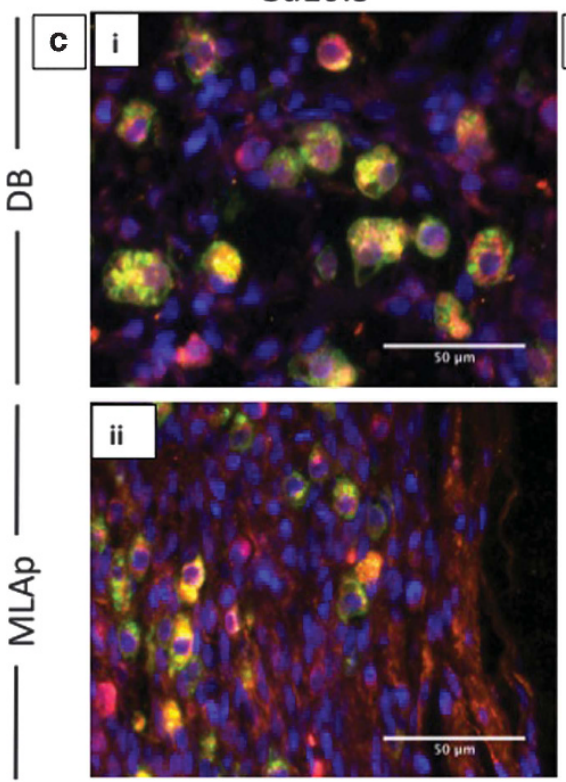

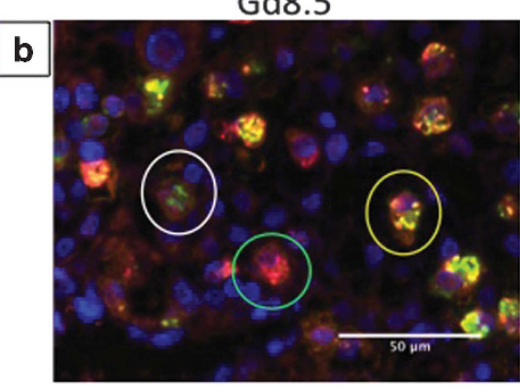

Gd12.5
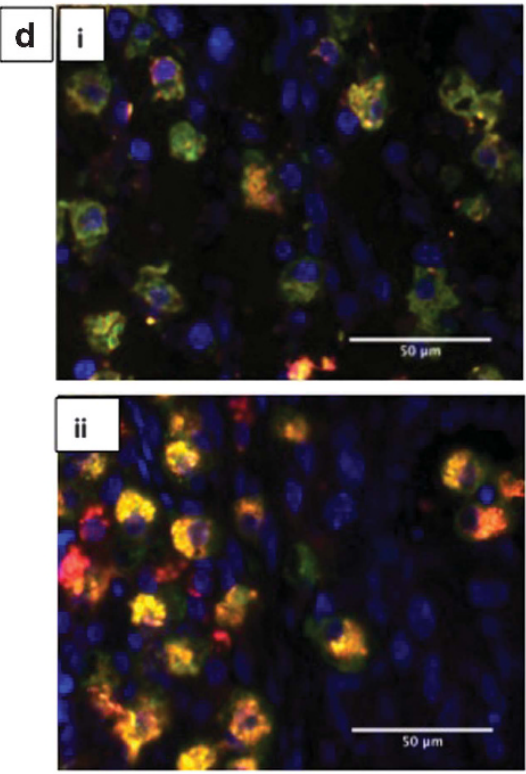

Figure 3 Perforin and granzyme A expression in Ncr1 ${ }^{\text {Gfp/Gfp }}$ DBA + uNK cells. Triple staining of $N c r 1^{G f p / G f p}$ uNK cells with DBA lectin (green), anti-PRF (red), anti-GZMA (orange), and DAPI (blue) at gd6.5 (a), 8.5 (b), 10.5 (c), and 12.5 (d). At gd6.5 (a), agranular DBA + uNK cells diffusely express lytic proteins. At gd8.5 (b), some granulated DBA + uNK cells in Ncr1 Gfp/Gfp mice display both PRF and GZMA expression (yellow circle), whereas others express solely PRF (green circle). In addition, a consistent proportion of DBA + uNK cells are unreactive for either lytic protein (white circle). At gd10.5 (c) in DB (i), many DBA + uNK cells express both lytic proteins or PRF singly. While PRF/GZMA co-expression is less common in MLAp (ii). At gd12.5 (d) uNK cells expressing lytic proteins are present in Ncr ${ }^{\text {Gfp/Gfp }}$ DB (i) and MLAp (ii) and occur in similar expression patterns. No DBA + uNK cells expressing only GZMA are observed at any gd studied. Images are representative of three replicate experiments using two implantation sites from three different pregnancies. DBA, dolichos biflorus agglutinin; DB, decidua basalis; gd, gestation day; GFP, green fluorescent protein; GZMA, granzyme A; MLAp, mesometrial lymphoid aggregate of pregnancy; PRF, perforin; NCR1, natural cytotoxicity receptor; uNK, uterine natural killer.

uNK cells are detected in high numbers in B6 mice at gd6.5 and 8.5 by WM-IHC. ${ }^{1}$ Proportionally, DBA + uNK cells in $N c r 1^{G f p / G f p} \mathrm{DB}$ are similar in frequency to those in B6 at gd6.5 $(P=0.261)$ and $8.5(P=0.207)$ but the $\mathrm{Ly} 49 \mathrm{C} / \mathrm{I}+$ subset is diminished (gd6.5: $17.3 \pm 3.1 \%$ vs. $32.7 \pm 3.1 \%$ in B6; $P=0.004$; gd8.5: $13.3 \pm 1.5 \%$ vs. $20.3 \pm 3.5 \%$ in $\mathrm{B} 6 ; P=0.034$ ) (Figure $5 \mathbf{a}, \mathbf{b})$. The proportions of both uNK cell subsets change drastically in B6 DB between gd6.5 and 8.5, with LY49C/I + (i.e., DBA-) uNK cells decreasing and DBA + uNK cells increasing. In $N c r 1^{G f p / G f p} \mathrm{DB}, \mathrm{DBA}+\mathrm{uNK}$ cell frequencies increased between gd6.5 and $8.5(P=0.038)$, but Ly49C/I + (DBA-) uNK cells were unchanged in frequency between these times $(P=0.112)$ (Figure 5a, b). Leukocytes other than NK cells are present in early decidua, therefore their frequencies were assessed. $\mathrm{CD} 8+\mathrm{T}$ cells, while not significantly different between genotypes at gd6.5 $(P=0.886)$ trended to more frequent at gd8.5 in $N c r 1^{G f p / G f p}$ than in $\mathrm{B} 6(11.7 \pm 3.2 \%$ vs.
$7.0 \pm 1.7 \% ; P=0.091) . \mathrm{CD} 4+\mathrm{T}$ cells were less frequent in $N c r 1^{G f p / G f p}$ than B6 at gd6.5 $(2.7 \pm 0.6 \%$ vs. $5.3 \pm 1.2 \%$; $P=0.023)$ and of similar frequency to B6 at gd8.5 (8.0 \pm $1.7 \%$ vs. $7.0 \pm 8.7 \% ; P=0.749)$, showing a significant increase between gd6.5 and $8.5(P=0.007)$. All other leukocyte populations were similar to B6 at gd6.5 and 8.5 (Figure 5a, b).

CD45 + /CD45 + conjugations. To determine whether loss of NCR1 expression altered the frequency of CD45 + leukocyte conjugate formation, WH-IHC was used to compare gd6.5 and gd8.5 Ncr1 ${ }^{G f p / G f p}$ to B6 implant sites. Fewer CD45 + /CD45 + conjugates occurred in $N c r 1^{G f p / G f p}$ than $\mathrm{B} 6 \mathrm{DB}$ at gd6.5 (5.2 $\pm 0.6 \%$ vs. $7.8 \pm 1.0 \% ; P=0.001)$ and gd8.5 $(11.7 \pm 1.4 \%$ vs. $19.0 \pm 1.8 \% ; P<0.001)$. As in $\mathrm{B} 6$ controls, a significant increase in the proportion of $N c r 1^{G f p / G f p} \mathrm{CD} 45+$ cells forming conjugates occurred between gd6.5 and $8.5 \quad(P=0.001)$ (Figure 5c). 


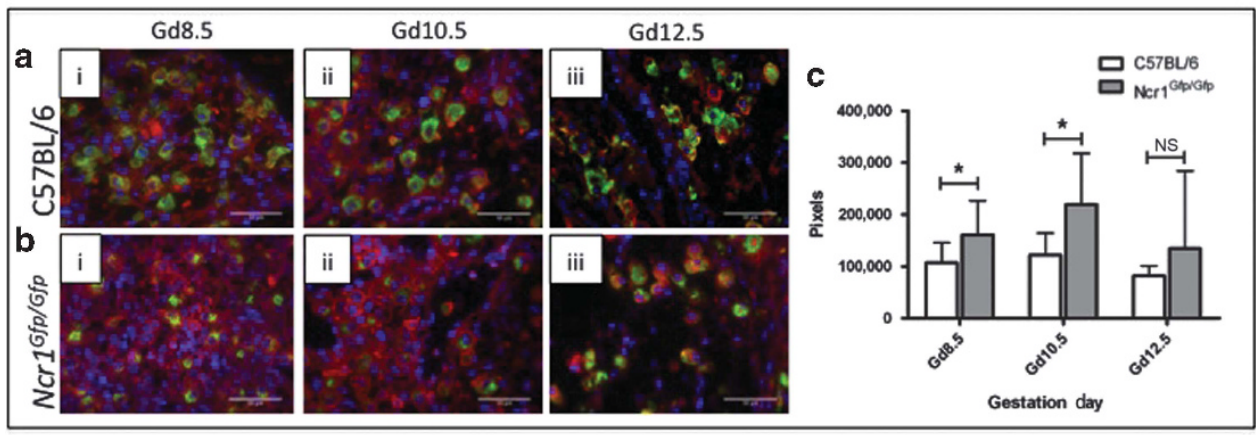

\section{PGF}

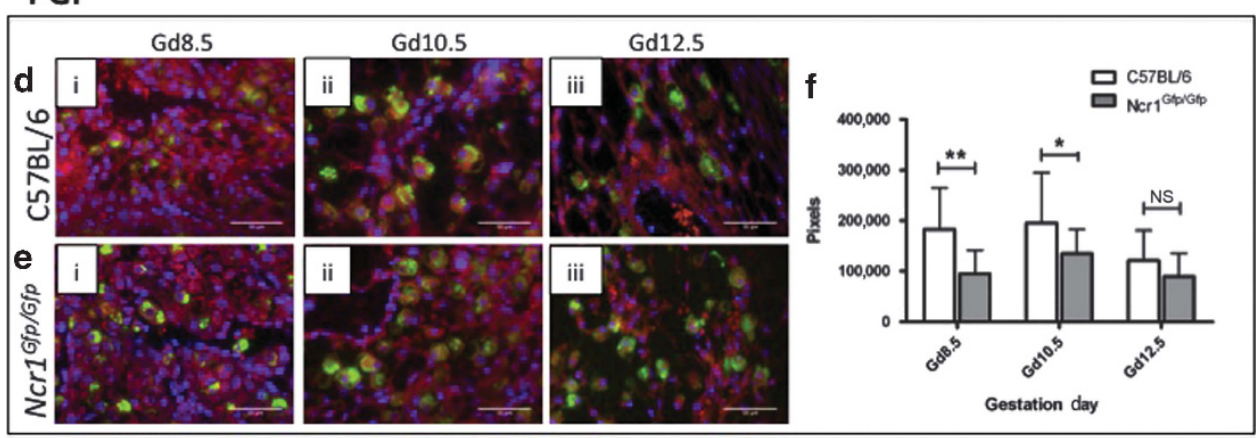

Figure 4 Immunoreactivity of VEGF and PGF in gd8.5-12.5 DBA + uNK cells. Representative images of VEGF staining in B6 (a) and Ncr1 Gfp/Gfp (b) decidua at gd8.5 (i), 10.5 (ii), and 12.5 (iii). (c) VEGF expression is significantly increased in Ncr1 ${ }^{\text {Gtp/Gfp }}$ uNK cells compared with B6 at gd8.5 and 10.5 . (d-e) Representative images of PGF staining in B6 (d) and Ncr1 $1^{G f p / G t p}$ (e) decidua at gd8.5 (i), 10.5 (ii), and 12.5 (iii). (f) PGF expression in DBA + uNK cells is significantly less in Ncr1 ${ }^{\text {Gfp/Gfp }}$ compared with B6 at gd8.5 and 10.5. Images are representative of three replicate experiments using two implantation sites from three independent pregnancies per gd per genotype. Data were assessed by an unpaired Student's $t$-test. ${ }^{\star} P<0.05$, ${ }^{\star *} P<0.01$, Data represent mean \pm s.d. DBA, dolichos biflorus agglutinin; gd, gestation day; GFP, green fluorescent protein; NCR1, natural cytotoxicity receptor; NS, not significant; PGF, placental growth factor; UNK, uterine natural killer; VEGF, vascular endothelial growth factor.

CD45 + /CD31 + conjugated cells. Gains in expression of CD31, an adhesion molecule expressed by endothelial cells, platelets, and immune cell subsets, ${ }^{31}$ were previously reported for gd6.5 B6 decidual CD $45+$ cells with $90 \%$ co-expression by gd8.5. ${ }^{1}$ Costaining of $N c r 1^{G f p / G f p}$ implant sites with anti-CD45 APC and anti-CD31 PE revealed fewer CD45 + leukocytes co-expressing CD31 than in B6 controls at gd6.5 $(P=0.033)$ and gd8.5 $(P=0.005)$ (Figure 5d). The proportion of CD31 $+\mathrm{CD} 45+$ cells increased statistically in Ncr1 $1^{G f p / G f p}$ but not B6 between gd6.5 and 8.5 (Figure 5d; $P=0.021$ ). When $N c r 1^{G f p / G f p} \mathrm{CD} 31+$ CD 45 + decidual cells were analysed by conjugation status, lower gd6.5 CD31 expression was attributed to less expression by nonconjugated CD $45+$ cells $(P=0.030$ vs. B6). Conjugated gd6.5 $\mathrm{CD} 31+\mathrm{CD} 45+$ cells were similar in frequency to B6 $(22.3 \pm 4.2 \%$ vs. $29.3 \pm 5.8 \%$ (B6); $P=0.164)$ (Supplementary Figure S3A). By gd8.5, proportions of conjugated and unconjugated $\mathrm{CD} 31+\mathrm{CD} 45+N c r 1^{G f p / G f p}$ cells were similar to $\mathrm{B} 6$ controls $(P=0.361$ and 0.334 , respectively). In addition, the proportion of conjugated $N c r 1^{G f p / G f p} \mathrm{CD} 31+$ cells in either homologous (CD31 + CD45 + /CD31 + CD45+) or heterologous $(\mathrm{CD} 31+\mathrm{CD} 45+/ \mathrm{CD} 31-\mathrm{CD} 45+)$ conjugates was similar to B6 $(P=0.165)$ (Supplementary Figure S3B).

DBA + uNK cell conjugations. Despite the loss of Ncr1, DBA + uNK cells formed conjugates at similar frequencies to those in B6 at gd6.5 and 8.5 (data not shown). Examination of leukocytes forming heterologous conjugates with $\mathrm{DBA}+\mathrm{uNK}$ cells indicated no differences in the composition of DBA + heterologous conjugates at gd6.5 or 8.5 (Supplementary Figure S3C, S3D). With the exception of CD4 $+\mathrm{T}$ cells, there was no change in $N c r 1^{G f p / G f p}$ DBA + leukocyte conjugate frequencies between gd6.5 and 8.5. CD4 + T cells had no participation in heterologous conjugates with DBA + uNK cells at gd6.5 but accounted for $11.6 \pm 4.6 \%$ of these conjugates at $\operatorname{gd} 8.5(P=0.012$ vs. $g \mathrm{~d} 8.5)$ (Supplementary Figure S3D).

Conjugates not involving uNK cells. Leukocytes were involved in heterologous conjugation events independent of DBA + uNK cells at both gd6.5 and 8.5. The only observed differences between $N c r 1^{G f p / G f p}$ and B6 mice in non-uNK cell leukocyte heterologous conjugate formation occurred in $\mathrm{CD} 11 \mathrm{c}+$ dendritic cells (DCs) at gd6.5. At this time, Ncr1 $1^{G f p / G f p}$ $\mathrm{CD} 11 \mathrm{c}+\mathrm{DCs}$ formed heterologous conjugates not involving $\mathrm{DBA}+\mathrm{uNK}$ cells at a frequency of $17.3 \pm 2.3 \%$ vs. $24.5 \pm 3.0 \%$ in B6 (Supplementary Figure S3E; $P=0.019$ ). Fewer non-uNK cell heterologous $\mathrm{CD} 4+$ and $\mathrm{CD} 8+\mathrm{T}$-cell conjugates were also observed in at gd6.5Ncr1 $1^{G f p / G f p}$, but these did not reach statistical significance $(\mathrm{CD} 4+: P=0.067 ; \mathrm{CD} 8+: P=0.078$ vs. B6). No differences were observed in types or frequency of 


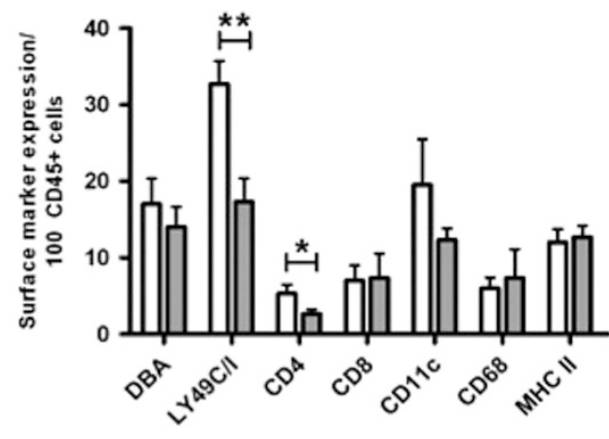

C

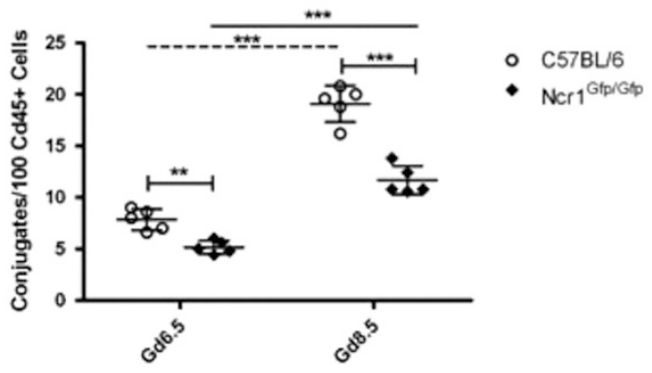

b

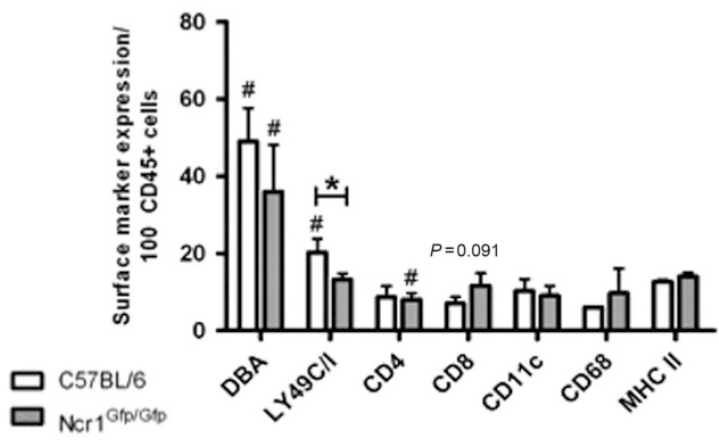

d

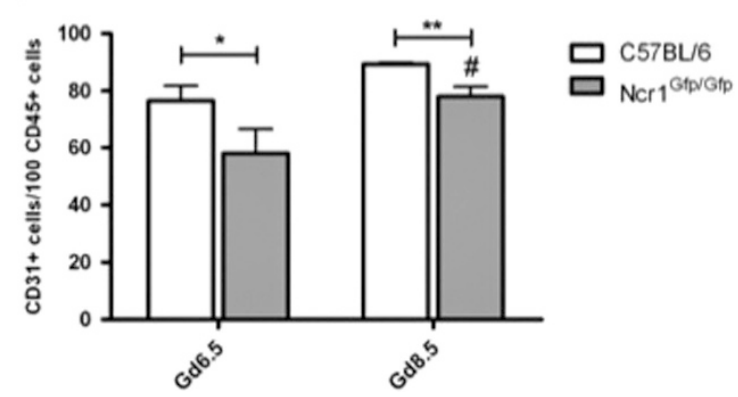

Figure 5 Leukocyte frequency, conjugate formation, and expression of activation markers. (a) Leukocyte populations at gd6.5 in B6 and Ncr1 Gfp/Gfp mice. Frequencies of $\mathrm{Ly} 49 \mathrm{C} / \mathrm{I}+\mathrm{uNK}$ cells and CD4 + T cells are lower in the absence of $N c r 1 .{ }^{\star} P<0.05,{ }^{\star \star} P<0.01 \mathrm{vs}$. B6. (b) At gd8.5, only Ly49C/I + uNK cells are affected by loss of Ncr1. DBA + uNK cells in both B 6 and $N c r 1^{\text {Gtp/Gfp }}$ mice increase from gd6.5 to 8.5 and Ly $49 \mathrm{C} / \mathrm{I}+\mathrm{uNK}$ cells decrease in frequency in B6. CD4 + T cells are unchanged in B6, but increase from gd6.5 to 8.5 in $N c r 1^{\text {Gfp/Gfp }}$ mice. ${ }^{*} P<0.05$ vs. B6; ${ }^{\#} P<0.05$ vs. gd6.5. (c) The overall proportion of CD $45+$ conjugates increases from gd6.5 to 8.5 in both mouse strains. Conjugate frequencies are lower in Ncr ${ }^{G t p / G f p}$ mice at both gd compared with controls. ${ }^{* \star} P<0.01$, ${ }^{\star \star \star} P<0.001$. (d) The proportion of CD45 + leukocytes with surface expression of the activation marker CD31 is significantly reduced by loss of Ncr1 at gd6.5 and 8.5. Co-expression of CD45 and CD31 is greater at gd8.5 than 6.5 in Ncr $1^{\text {Gfp/Gfp }}$ mice, but no difference between gd in B6 mice. ${ }^{*} P<0.05$, ${ }^{*} P<0.01$ vs. B6; ${ }^{*} P<0.05$ vs. gd6.5. Images are representative of three replicate experiments using two implantation sites from three independent pregnancies per gd per genotype. Data represent mean \pm s.d. For statistical analysis data were analyzed by one-way ANOVA with post hoc Tukey's multiple comparison test. The data shown here as two separate graphs (a and $\mathbf{b}$ ) were grouped prior to analysis. ANOVA, analysis of variance; DBA, dolichos biflorus agglutinin; gd, gestation day; GFP, green fluorescent protein; NCR1, natural cytotoxicity receptor; uNK, uterine natural killer.

non-uNK cell heterologous conjugate formation between strains at gd8.5 (Supplementary Figure S3E, S3F).

\section{DISCUSSION}

This study builds from an initial report that documented biased expression of NCR1 by DBA $+\mathrm{uNK}$ cells and reported essential roles for NCR1 in murine pregnancy. ${ }^{20}$ In the initial study, normal numbers of uNK cells were described in $N c r 1^{G f p / G f p}$ mice, but they were smaller than in gestation stage-matched control mice. Early $N c r 1^{G f p / G f p}$ implant sites displayed limited angiogenesis and mildly delayed embryonic development, whereas midgestation sites lacked physiological spiral artery modification. ${ }^{20}$ These features were accompanied by increased fetal resorption rates but did not limit overall pregnancy success in breeding pairs. To further understand the importance of NCR1 in uNK cell maturation, function and promotion of pregnancy success, in depth studies were undertaken. NCR1 (NKp46) is a highly conserved transmembrane receptor specific to NK cells and is expressed at all stages of mouse and human NK cell development. ${ }^{32,33}$ The gestationally unique, angiogenesis-promoting $\mathrm{DBA}+\mathrm{uNK}$ cell subset expresses eight-fold more Norl RNA than the splenic-
NK cell-like DBA- uNK cell subtype, ${ }^{20}$ thus our study focused on the DBA + uNK cell subset.

Morphological assessments of uNK cell maturity employ cell diameter, cytoplasmic granule number, and nuclear morphology. ${ }^{4} \mathrm{Ncr} 1^{\mathrm{Gfp} / \mathrm{G} f \mathrm{p}}$ mice had higher proportions of immature uNK cell subtypes across early and midpregnancy than gd-matched controls. This was particularly apparent at gd12.5 when uNK cells in $\mathrm{DB}$ of control mice are fully mature, senescent cells thought to be stationary, cytokine secreting cells proceeding to apoptosis. $^{29,34}$ In Ncr $1^{G f p / G f p}$, the most immature Type I agranular uNK cells persisted to gd12.5 and fully mature Type IV uNK cells were infrequent. These data support the conclusion derived from cell diameter measurements that NCR1 participates in the stepwise maturation of uNK cells. As Type IV uNK cells are observed in $N c r 1^{G f p / G f p} \mathrm{DB}$, their diminished numbers are likely due to maturation delay and overall uNK cell immaturity than to an incapacity for terminal differentiation. Human pNK cells acquire NKp46 after CD56 and constitutive NKp46 expression occurs in NK cells in lymph node, lung, liver, and blood. ${ }^{32,35}$ UNK cells present in late menstrual cycle endometrium ${ }^{36}$ and early decidua $^{28}$ also constitutively express the receptor. Thus, NKp46 may contribute to human uNK cell maturation. 
Ultrastructural studies identified biosynthetic roles for NCR1 within uNK cells. Although $N c r 1^{G f p / G f p}$ uNK cells contained smaller but structurally typical granules at gd6.5 and 8.5, uNK cells at later gd contained atypical granules, ${ }^{9,29,37}$ although granule number per cell did not differ from controls. Granule functionality was assessed by immunoreactivity quantification of two granule-associated proteins, PRF and GZMA. At gd6.5 and 8.5, when granules are still developing, $\mathrm{Ncr}^{\mathrm{Gfp} / \mathrm{Gfp}}$ decidua contained fewer uNK cells coexpressing PRF and GZMA and induction of GZMA synthesis appeared to be delayed as uNK cells solely expressing PRF were at a much higher frequency than in controls. At gd10.5 when ultrastructural differences were apparent between $N c r 1^{G f p / G f p}$ and B6 granules, GZMA expression by $\mathrm{Ncr}^{\mathrm{Gfp} / \mathrm{Gfp}} \mathrm{uNK}$ cells was also significantly lower (Table 1). Acquisition of lytic granules transitions NK cells from immature to mature, and occurs in uNK cells despite their limited lytic function. ${ }^{9}$ Thus, these studies reinforce the conclusion drawn from the morphological subtyping that DBA $+N c r 1^{G f p / G f p}$ uNK cells are immature. In cytotoxic pNK cells, PRF enables GZMA-mediated apoptosis ${ }^{38}$ and during pregnancy PRF induction in uNK cells occurs as a result of maternal stimulation independent of circulating pregnancy hormones. ${ }^{39}$ Identity of the maternal factors responsible for PRF induction remains unknown but treatment of first trimester human uNK cells with decidual stromal cell-derived IL-33 significantly attenuated uNK cell cytotoxicity and decreased the expression of the activating receptors NKp30 and NKG2D (NKp46 expression was not measured). ${ }^{40}$ Gains in NKp30 and NKp46 expression by human NK cell precursors increases NK cell cytolytic activity. ${ }^{35}$ Together these data implicate local paracrine signals from maternal decidua and NCRs in acquisition of lytic proteins by NK cells, including lytically inert uNK cells.

DBA + uNK cells are potent regulators of early decidual vascular development, ${ }^{8,13}$ producing angiokines, contributing to nascent blood vessel pruning, ${ }^{41}$ and modifying existing maternal arteries, ${ }^{14}$ VEGF and PGF, hallmark uNK cell products, were examined to address whether NCR1 signaling is required for angiokine synthesis. A reciprocal impact of elevated VEGF and reduced PGF was identified that may reflect changes in synthesis rather than altered consumption, however the latter cannot be ruled out as components of the VEGFR signaling family were not studied. Ncr $1^{G f p / G f p}$ mice with significantly reduced PGF production have impairments in decidual angiogenesis ${ }^{20}$ similar to mice lacking $\mathrm{PGF}^{42}$ suggesting that maternal VEGF is unable to substitute for PGF in normal implantation sites. This may be relevant to the pathophysiology of PGF-deficient human preeclampsia. ${ }^{43}$ PanLy49 receptor-deficient mice also show impairments in uNK cell maturation and decidual vasculogenesis resembling $N c r 1^{G f p / G f p}$, however, VEGF expression is reduced in this strain. ${ }^{16}$ Multiple reports suggest that PGF stimulates VEGF activity, ${ }^{44,45}$ but PGF can also antagonize VEGF activity when it is co-produced by single cells. ${ }^{46}$ The differences in regulation of VEGF suggest a complexity that requires future study to better understand the regulatory pathways controlling angiokine production and secretion by uNK cells.

Prior to immune synapse formation between NK cells and target cells, NKp46 (NCR1) is uniformly distributed on human NK cell membranes. After target cell contact, NKp46 segregates rapidly into large clusters at the point of contact, which affects cytoskeletal rearrangement, influences F-actin assembly, and directs granule polarization. ${ }^{33}$ Human uNK cells in contrast form immature synapses that fail to polarize granules to the synapse. ${ }^{11}$ Interestingly, of the NCRs, only NKp46 is able to induce granule polarization in human uNK cells upon receptor ligation. ${ }^{17}$ These studies suggested that uNK cell conjugate formation might be impaired in NCR1-deficient mice. Indeed, significantly fewer CD45 +/CD45 + conjugates were present at gd6.5 and 8.5 in $N c r 1^{G f p / G f p}$ decidua, but the proportion and composition of DBA + cell conjugates was not significantly altered from controls.

At gd6.5, the proportion of CD11c + DCs forming heterologous conjugates that did not include DBA + uNK cells fully accounted for the overall deficits in CD45 + /CD45 + conjugate formation. Although these conjugates likely included DBA$(\mathrm{Ly} 49 \mathrm{C} / \mathrm{I}+)$ uNK cells, their numbers are unlikely to be affected as Ncrl is expressed at low levels on DBA- uNK cells, ${ }^{20}$ and no alterations were observed in $N c r 1^{G f p / G f p} \mathrm{CD} 11 \mathrm{c}+/ \mathrm{DBA}+$ heterologous conjugates. Further, NKp30, rather than NKp46 or NKp44 is involved in human NK cells' lytic recognition of $\mathrm{DCs}^{47}$ and regulatory synapses reported at the human maternalfetal interface, involve DC-SIGN on DCs and ICAM-3 on uNK cells. ${ }^{48}$ Thus, none of the known DC-NK cell interactions predict NCR1 usage in mouse DC-NK conjugation and suggest that DC interactions with non-uNK cell lineages are reduced in $N c r 1^{G f p / G f p}$.

This study highlights the importance of NCR1 for uNK cell maturation and function. Although many studies of uNK cells in human pregnancy complications focus on interactions between mismatched maternal killer immunoglobulin-like receptors and fetal HLA molecules, ${ }^{15}$ our in vivo work along with that of others investigating NCRs on human uNK cells in culture highlights the significance of MHC-independent receptors in murine pregnancy and predicts that this receptor family has important roles in human pregnancy.

\section{METHODS}

Mice. Heterozygous Ncr 1 loss of function breeding pairs on a C57BL/6 (B6) background (UkCa:B6.Ncr1 ${ }^{+/ G f p}\left(\mathrm{Ncr} 1^{+/ G f p)}\right)^{23}$ were provided by Dr O. Mandelboim (Hebrew University-Hadassah Medical School, Jerusalem, Israel, through the laboratory of Dr F. Colucci (University of Cambridge, Cambridge UK)), bred to homozygosity $\left(N c r 1^{G f p / G f p}\right)$ at Queen's University and housed in a monitored, pathogen-free environment. Control B6 males and females were purchased at 7 weeks of age from Charles River Laboratories (St-Constant, QU, Canada). B6 or Ncr $1^{\text {Gfp/Gfp }}$ studs were mated to B6 or $N c r 1^{G f p / G f p}$ females, respectively. Matings were timed from copulation plug detection (gd0.5); euthanasia was by cervical dislocation at gd6.5, 8.5, 10.5, or 12.5. Mouse handling was in accordance with the guidelines of the Canadian Council on Animal Care and conducted under animal care protocols approved by Queen's University. 
Immunostaining and morphometry. Implantation sites from gd6.5 to $12.5 \mathrm{Ncr} 1^{G f p / G f p}$ and B6 mice were dissected, immersion fixed in $4 \%$ paraformaldehyde, processed, and paraffin embedded. At least two healthy implantation sites from each pregnancy $(n=3$ pregnancies per genotype or 6 specimens per genotype) were cut as serial sections of $6 \mu \mathrm{m}$ and mounted onto glass slides. Each staining procedure (outlined below) was conducted as three replicate experiments. Stained slides were viewed with a Zeiss M1 imager (Zeiss, Toronto, ON, Canada) and photographed using Axiovision 4.8 software (Zeiss, Toronto, ON, Canada). Morphometric analyses were undertaken by a single blinded observer (AMF) using serial sections at least $42 \mu \mathrm{m}$ apart to avoid duplicate enumeration of uNK cells.

(i) DBA lectin staining: Staining for DBA lectin-reactive uNK cells used published protocols. ${ }^{4}$ DBA + uNK cells were scored for maturation stage using previously published criteria involving diameter, cytoplasmic granule number, and nuclear morphology. ${ }^{4}$ All nucleated uNK cells not residing in vascular spaces were enumerated and classified for maturity in at least five images captured at $\times 400$ magnification of DB (gd6.5-12.5) and/or MLAp (gd10.512.5) per specimen ( $n=6$ specimens per gd per genotype).

(ii) Immunofluorescence for $u N K$ cell products: Deparaffinized sections were pre-treated by heat-activated antigen retrieval (TrisEDTA (10 mM) pH9.0 for VEGF, PRF, GZMA; Citrate buffer (10 mM) pH 6.0 for PGF) for 5-10 min. Sections were blocked with 1\% PBS-BSA for $30 \mathrm{~min}$ at room temperature and subsequently incubated either overnight at $4{ }^{\circ} \mathrm{C}$ with $5 \mu \mathrm{g} \mathrm{ml}^{-1}$ rabbit anti-mouse VEGFA (Abcam, Toronto, ON, Canada; ab46154) or $40 \mu \mathrm{g} \mathrm{ml}^{-1}$ rat anti-mouse PRF (Abcam; ab16074) and $4 \mu \mathrm{g} \mathrm{ml}^{-1}$ goat anti-mouse GZMA (Santa Cruz Biotech, Dallas, TX; sc5510), or for $2 \mathrm{~h}$ (room temperature) with $5 \mu \mathrm{g} \mathrm{ml}^{-1}$ rabbit anti-PGF (Abcam; ab9542). Fluorescently conjugated secondary antibodies $\left(10 \mu \mathrm{g} \mathrm{ml}^{-1}\right.$ goat anti-rabbit Alexa fluor 594 (ThermoFisher, Eugene, OR, USA; A-11012), $10 \mu \mathrm{g} \mathrm{ml}^{-1}$ goat anti-rat Alexa Fluor 594 (Abcam; ab150160), or $7.5 \mu \mathrm{g} \mathrm{ml}^{-1}$ donkey anti-goat Alexa Fluor 647 (Jackson ImmunoResearch, West Grove, PA; 705-605147) and $10 \mu \mathrm{g} \mathrm{ml}^{-1}$ DBA lectin FITC (Vector Laboratories, Burlingame, CA; FL-1031)) were then added ( $1 \mathrm{~h}$, room temperature). The nuclei were visualized by DAPI staining with ProLong Gold antifade reagent with DAPI (ThermoFisher). For VEGF and PGF staining, the multidimensional acquisition feature in Axiovision 4.8 software was used to set manual exposure times for DAPI and DBA lectin, whereas VEGF/PGF was set to auto-exposure, thereby producing an unbiased image based on software-detected staining intensity. Ten images captured at $\times 400$ magnification $(n=6$ specimens per gd per genotype) were analyzed for pixel intensity using GNU Image Manipulation Program (GIMP 2.8.10 software, Gimp.org). For PRF and GZMA dual staining, at least seven images captured at $\times 400$ magnification were analyzed for expression in all uNK cells per field of view. DBA + granules were also scored in each nucleated, granulated uNK cell ( $n=6$ specimens per gd per genotype).

Ultrastructure. For electron microscopy, $1 \mathrm{~mm}^{3}$ pieces of gd6.5, 8.5 , and $10.5 \mathrm{Ncr} 1^{\mathrm{G} f \mathrm{p} / \mathrm{G} f \mathrm{p}}$ and B6 DB $(n=3$ litters per genotype with at least two implantation sites from each litter) were immersion fixed (2\% paraformaldehyde, $2.5 \%$ glutaraldehyde, $24-48 \mathrm{~h}$ ). Post-fixation tissues were washed in $70 \%$ ethanol and incubated ( $1 \mathrm{~h}$ room temperature) in $1 \%$ osmium tetroxide, then dehydration and embedding procedures were performed following manufacturer's recommendations (Embed 812 resin; Electron Microscopy Science-EMS). Semi-thin sections $(1 \mu \mathrm{m})$ were stained with toluidine blue and ultra-thin sections were contrasted with uranyl acetate and lead citrate; micrographs were taken on a Hitachi H-7000 transmission electron microscope (Hitachi, Tokyo, Japan).

Whole-mount in situ immunohistochemistry. Gd6.5 and 8.5 implantation sites from at least three different $N c r 1^{G f p / G f p}$ litters per gd were studied using WM-IHC as previously described. ${ }^{2}$ Any small, abnormally pale, or resorbing implantation sites were excluded. Under microscopic magnification, separated implantation sites were incised antimesometrially and myometrium was retracted away. Each decidual capsule was then bisected midsagittally using a scalpel blade and incubated in $200 \mu \mathrm{l}$ PBS- $1 \%$ BSA- $0.1 \%$ sodium azide (PBA) for $1 \mathrm{~h}$ with $10 \mu \mathrm{g} \mathrm{ml}^{-1}$ of blocking antibody to the IgG Fc receptor (antiCD16/CD32; supernatant of hybridoma 2.4G2, ATTC, Manassas, VA, USA) and $2-10 \mu \mathrm{g} \mathrm{ml}^{-1}$ of up to three differently conjugated fluorescent primary antibodies (Supplementary Table S1). For DBA lectin staining, implantation sites were transferred to $200 \mu \mathrm{l}$ fresh $\mathrm{PBA}$, and incubated for an additional $10 \mathrm{~min}$ with $30 \mu \mathrm{g} \mathrm{ml} \mathrm{m}^{-1}$ FITC- or $15 \mu \mathrm{g} \mathrm{ml}^{-1}$ TRITC-conjugated DBA lectin (Supplementary Table S1). Supplementary Table S2 presents the antibody combinations used. Labeling was terminated by addition of $1 \mathrm{ml}$ PBA. The intact, live tissue was placed on a microscope slide with the cut surface facing upward, coverslipped, viewed by epifluorescence microscopy, and photographed using an AxioCam-equipped Zeiss M1 imager with Axiovision 4.8 software. Different antibody staining combinations were used in studies of littermates.

Conjugate assessment. Leukocyte conjugates were analyzed using 10 epifluorescence images captured at $\times 200$ magnification. Leukocyteleukocyte pairs were scored as described in Felker and Croy. ${ }^{1}$ Briefly conjugation was scored when two CD45 + cells were observed in close contact in the same plane of focus with a slight flattening of both cells' membranes at the point of contact. At least $50 \mathrm{CD} 45+$ conjugates were counted for each antibody combination in two implantation sites from three different litters. Each cell of the pair was scored for antibody reactivity. Conjugates were scored as homologous when the two CD45 + cells shared expression of surface marker under study or heterologous when the two CD45 + cells expressed dissimilar surface markers.

Statistical analyses. Statistical significance for immunofluorescence analyses and conjugated cell counts was assessed by Student's $t$-test or one-way analysis of variance with post hoc Tukey's multiple comparison test using Prism5 Software (GraphPad Software, La Jolla, CA). $P<0.05$ was considered significant. Data are expressed as mean \pm s.d.

SUPPLEMENTARY MATERIAL is linked to the online version of the paper at http://www.nature.com/mi

\section{ACKNOWLEDGMENTS}

We thank Professor Aureo Yamada, Dr Patricia Lima, Dr Matthew Rätsep, and Ms Mackenzie Redhead for helpful discussions and Dr Xiaohu Yan for assistance in the ultrastructural studies. This work was supported by awards from Queen's University (A.M.F.) and NSERC, CFI, and the Canada Research Chairs Program (B.A.C.).

\section{AUTHOR CONTRIBUTIONS}

A.M.F. conducted all experiments, analyzed the data, and wrote and edited the manuscript. B.A.C. designed the experiments and contributed to the writing and editing of the manuscript.

\section{DISCLOSURE}

The authors declared no conflict of interest.

c 2017 Society for Mucosal Immunology

\section{REFERENCES}

1. Felker, A.M. \& Croy, B.A. Uterine natural killer cell partnerships in early mouse decidua basalis. J. Leukoc. Biol. 100, 645-655 (2016).

2. Croy, B. A., Chen, Z., Hofmann, A. P., Lord, E. M., Sedlacek, A. L. \& Gerber, S. A. Imaging of vascular development in early mouse decidua and its association with leukocytes and trophoblasts. Biol. Reprod. 87, 125. $1-125,11$ (2012)

3. Vacca, P., Mingari, M.C. \& Moretta, L. Natural killer cells in human pregnancy. J. Reprod. Immunol. 97, 14-19 (2013).

4. Paffaro, A., Bizinotto, C., Joazeiro, P. \& Yamada, T. Subset classification of mouse uterine natural killer cells by DBA lectin reactivity. Placenta $\mathbf{2 4}$, 479-488 (2003). 
5. Sojka, D.K. et al. Tissue-resident natural killer (NK) cells are cell lineages distinct from thymic and conventional splenic NK cells. Elife 3, e01659 (2014).

6. Redhead, M.L., Portilho, N.A., Felker, A.M., Mohammad, S., Mara, D.L. \& Croy, B.A. The transcription factor NFIL3 is essential for normal placental and embryonic development but not for uterine natural killer (UNK) cell differentiation in mice. Biol. Reprod. 94, 101 (2016).

7. Parr, E.L., Young, L.H., Parr, M.B. \& Young, J.D. Granulated metrial gland cells of pregnant mouse uterus are natural killer-like cells that contain perforin and serine esterases. J. Immunol. 145, 23652372 (1990).

8. Hanna, J. et al. Decidual NK cells regulate key developmental processes at the human fetal-maternal interface. Nat. Med. 12, 1065-1074 (2006).

9. Lima, P. D., Croy, B. A., Degaki, K. Y., Tayade, C. \& Yamada, A. T. Heterogeneity in composition of mouse uterine natural killer cell granules. J. Leukoc. Biol. 92, 195-204 (2012).

10. Jabrane-Ferrat, N. \& Siewiera, J. The up side of decidual natural killer cells: new developments in immunology of pregnancy. Immunology 141, 490-497 (2014).

11. Kopcow, H.D. et al. Human decidual NK cells form immature activating synapses and are not cytotoxic. Proc. Natl. Acad. Sci. USA 102, 15563-15568 (2005).

12. Zhang, J., Yamada, A.T. \& Croy, B.A. DBA-lectin reactivity defines natural killer cells that have homed to mouse decidua. Placenta 30, 968-973 (2009).

13. Chen, Z. et al. DBA-Lectin reactivity defines mouse uterine natural killer cell subsets with biased gene expression. Biol. Reprod. 87, 81 (2012).

14. Ashkar, A.A. \& Croy, B.A. Functions of uterine natural killer cells are mediated by interferon gamma production during murine pregnancy. Semin. Immunol. 13, 235-241 (2001).

15. Madeja, Z. et al. Paternal MHC expression on mouse trophoblast affects uterine vascularization and fetal growth. Proc. Natl. Acad. Sci. USA 108, 4012-4017 (2011).

16. Lima, P.D., Tu, M.M., Rahim, M.M., Peng, A.R., Croy, B. \& Makrigiannis, A.P. Ly49 receptors activate angiogenic mouse DBA(+) uterine natural killer cells. Cell Mol. Immunol. 11, 467-476 (2014).

17. Siewiera, J. et al. Natural cytotoxicity receptor splice variants orchestrate the distinct functions of human natural killer cell subtypes. Nat. Commun. 6, 10183 (2015).

18. Biassoni, R., Pessino, A., Bottino, C., Pende, D., Moretta, L. \& Moretta, A. The murine homologue of the human nkp46, a triggering receptor involved in the induction of natural cytotoxicity. Eur. J. Immunol. 29, 1014-1020 (1999).

19. Manaster, I. \& Mandelboim, O. The unique properties of uterine NK cells. Am. J. Reprod. Immunol. 63, 434-444 (2010).

20. Felker, A.M., Chen, Z., Foster, W.G. \& Croy, B.A. Receptors for non-mhc ligands contribute to uterine natural killer cell activation during pregnancy in mice. Placenta 34, 757-764 (2013).

21. Boerman, G.H. et al. Role of NKG2D, DNAM-1 and natural cytotoxicity receptors in cytotoxicity toward rhabdomyosarcoma cell lines mediated by resting and il-15-activated human natural killer cells. Cancer Immunol. Immunother. 64, 573-583 (2015).

22. Chisholm, S.E., Howard, K., Gómez, M.V. \& Reyburn, H.T. Expression of ICPO is sufficient to trigger natural killer cell recognition of herpes simplex virus-infected cells by natural cytotoxicity receptors. J. Infect. Dis. 195, 1160-1168 (2007).

23. Gazit, R. et al. Lethal influenza infection in the absence of the natural killer cell receptor gene ncr1. Nat. Immunol. 7, 517-523 (2006).

24. Satoh-Takayama, N. et al. Microbial flora drives interleukin 22 production in intestinal nkp46 + cells that provide innate mucosal immune defense. Immunity 29, 958-970 (2008).

25. Gur, C. et al. Recognition and killing of human and murine pancreatic beta cells by the NK receptor nkp46. J. Immunol. 187, 3096-3103 (2011).

26. Giuliani, E., Parkin, K.L., Lessey, B.A., Young, S. L. \& Fazleabas, A. T. Characterization of uterine NK cells in women with infertility or recurrent pregnancy loss and associated endometriosis. Am. J. Reprod. Immunol. 72, 262-269 (2014).
27. Chiba, H., Fukui, A., Fuchinoue, K., Funamizu, A., Tanaka, K. \& Mizunuma, $H$. Expression of natural cytotoxicity receptors on and intracellular cytokine production by NK cells in women with gestational diabetes mellitus. Am. J. Reprod. Immunol. 75, 529-538 (2016).

28. El Costa, H. et al. Critical and differential roles of nkp46- and nkp30activating receptors expressed by uterine NK cells in early pregnancy. J. Immunol. 181, 3009-3017 (2008).

29. Delgado, S.R., McBey, B.A., Yamashiro, S., Fujita, J., Kiso, Y. \& Croy, B.A. Accounting for the peripartum loss of granulated metrial gland cells, a natural killer cell population, from the pregnant mouse uterus. J. Leukoc. Biol. 59, 262-269 (1996).

30. Croy, B.A. \& Kiso, Y. Granulated metrial gland cells: a natural killer cell subset of the pregnant murine uterus. Microsc. Res. Tech. 25, 189-200 (1993).

31. Kishore, M., Ma, L., Cornish, G., Nourshargh, S. \& Marelli-Berg, F.M. Primed T cell responses to chemokines are regulated by the immunoglobulin-like molecule CD31. PLoS One 7, e39433 (2012).

32. Walzer, T. et al. Identification, activation, and selective in vivo ablation of mouse NK cells via nkp46. Proc. Natl. Acad. Sci. USA 104, 3384-3389 (2007).

33. Hadad, U., Thauland, T.J., Martinez, O.M., Butte, M.J., Porgador, A. \& Krams, S. M. NKp46 clusters at the immune synapse and regulates NK cell polarization. Front. Immunol. 6, 495 (2015).

34. Rajagopalan, S. HLA-G-mediated NK cell senescence promotes vascular remodeling: implications for reproduction. Cell Mol. Immunol. 11, 460-466 (2014).

35. Sivori, S. et al. Early expression of triggering receptors and regulatory role of 2B4 in human natural killer cell precursors undergoing in vitro differentiation. Proc. Natl. Acad. Sci. USA 99, 4526-4531 (2002).

36. Male, V., Hughes, T., McClory, S., Colucci, F., Caligiuri, M. A. \& Moffett, A. Immature NK cells, capable of producing IL-22, are present in human uterine mucosa. J. Immunol. 185, 3913-3918 (2010).

37. Stewart, I. \& Peel, S. The structure and differentiation of granulated metrial gland cells of the pregnant mouse uterus. Cell Tissue Res. 184, 517-527 (1977).

38. Voskoboinik, I., Whisstock, J.C. \& Trapani, J.A. Perforin and granzymes: function, dysfunction and human pathology. Nat. Rev. Immunol. 15, 388-400 (2015).

39. Zheng, L.M., Joag, S.V., Parr, M.B., Parr, E.L. \& Young, J.D. Perforinexpressing granulated metrial gland cells in murine deciduoma. J. Exp. Med. 174, 1221-1226 (1991).

40. Hu, W.T., Huang, L.L., Li, M.Q., Jin, L.P., Li, D.J. \& Zhu, X.Y. Decidual stromal cell-derived IL-33 contributes to th2 bias and inhibits decidual NK cell cytotoxicity through nf- $\mathrm{kb}$ signaling in human early pregnancy. J. Reprod. Immunol. 109, 52-65 (2015).

41. Hofmann, A.P., Gerber, S.A. \& Croy, B.A. Uterine natural killer cells pace early development of mouse decidua basalis. Mol. Hum. Reprod. 20, 66-76 (2014).

42. Rätsep, M.T., Carmeliet, P., Adams, M.A. \& Croy, B.A. Impact of placental growth factor deficiency on early mouse implant site angiogenesis. Placenta 35, 772-775 (2014).

43. Staff, A.C. et al. Redefining preeclampsia using placenta-derived biomarkers. Hypertension 61, 932-942 (2013).

44. Park, J.E., Chen, H.H., Winer, J., Houck, K.A. \& Ferrara, N. Placenta growth factor. Potentiation of vascular endothelial growth factor bioactivity, in vitro and in vivo, and high affinity binding to flt-1 but not to flk-1/KDR. J. Biol. Chem. 269, 25646-25654 (1994).

45. Marcellini, M. et al. Increased melanoma growth and metastasis spreading in mice overexpressing placenta growth factor. Am. J. Pathol. 169, 643-654 (2006).

46. Eriksson, A. et al. Placenta growth factor-1 antagonizes vegf-induced angiogenesis and tumor growth by the formation of functionally inactive plgf-1/NEGF heterodimers. Cancer Cell 1, 99-108 (2002).

47. Mace, E.M. \& Orange, J.S. Multiple distinct NK-cell synapses. Blood 118, 6475-6476 (2011).

48. Dietl, J., Hönig, A., Kämmerer, U. \& Rieger, L. Natural killer cells and dendritic cells at the human feto-maternal interface: an effective cooperation?. Placenta 27, 341-347 (2006). 